\title{
Call blocking performance for dynamic channel allocation technique in future mobile satellite systems
}

\author{
E.Del Re \\ R. Fantacci \\ G. Giambene
}

Indexing tems: Low earth orbit systems, Mobile satellite systems, Personal communications, Intersatellite handover

\begin{abstract}
Different dynamic channel allocation (DCA) approaches based on the evaluation of a cost function are proposed. The scenario considered is low earth orbit and geostationary orbit mobile satellite systems. A suitable user mobility model has been defined to generate interbeam handover requests. Different alternatives to manage interbeam handovers have been investigated. Among them, the most promising solution seems to be the queuing of handover requests. The quality of service parameters that have been considered are: blocking probability for new call arrivals, handover failure probability and the probability of incompletely served call owing to the initial blocking or to the failure of a subsequent handover request. Comparisons among the proposed DCA techniques and the fixed channel allocation technique have been carried out to find a solution that represents a good trade-off between the blocking performance and the required signalling load.
\end{abstract}

\section{Introduction}

The steady growth in the cellular telephony market requires an ever-increasing capacity to meet the range of services and the foreseen wide population of users of the future personal communication services (PCS). PCS is an extension and integration of existing and future wireless and wired communication networks into a common framework, ultimately allowing the communication with a user anywhere and anytime, regardless of the terminal and using a universal personal identification number $[1,2]$. Therefore the third-generation cellular system, namely PCS, will encompass in a unique standard the different technologies of cordless, terrestrial and satellite mobile cellular networks.

In this paper, a future integrated scenario is

(C) IEE, 1996

IEE Procecdings online no. 19960742

Paper first received 20th June 1995 and in revised form 16th May 1996

The authors are with the Dipartimento di Ingegneria Elettronica, Università degli Studi di Firenze, Via S. Marta, 3, 50139, Firenze, Italy considered, where terrestrial and satellite systems will harmonise to offer a global high-quality coverage. Various integrated solutions have been proposed in the literature. In this paper, we refer to the ultimately and more complex level of integration for future PCS, named 'system integration' [3, 4]:

- satellite and terrestrial networks belong to a unique system

- the same techniques as those of the terrestrial system (multiaccess scheme, protocols for channel allocation and mobility management, etc ...) are adopted for the satellite system with a considerable technology reuse for the common parts (except RF equipment). The frequency bands allocated to the terrestrial segment (UHF band) and to the satellite segment ( $\mathrm{L}$ and $\mathrm{S}$ bands) will be different in a future PCS.

- the use of dual-mode mobile terminals allows automatic rerouting procedures between terrestrial and satellite networks (i.e. internetwork handovers).

In an integrated scenario mobile satellite systems (MSSs) will play a fundamental role in providing services to scarcely populated areas (the satellite network complements the terrestrial one) or to congested areas (the satellite network acts as a back-up system). At present, R\&D efforts are addressed towards the definition of MSSs that use a constellation of nongeostationary orbit (NGSO) satellites. In particular, low earth orbit mobile satellite systems (LEOMSSs) are considered since they permit to relax the constraints on the link budget and allow the use of low-power hand-held mobile terminals. Several LEOMSSs have been proposed, e.g. Iridium (by Motorola), Aries (by Constellation Communications Inc.), Globalstar (by Loral Qualcomm Satellite Services Inc.), Teledesic (the former Calling, by Microsoft et $a l$.). The working example assumed in this paper is given by the Iridium system; however, the techniques described here can be directly applied also to other satellite orbital configurations. The Iridium system considered in this paper is formed by 66 satellites over six polar circular orbits at about $780 \mathrm{~km}$ of altitude $[5$, 6]. Each satellite covers a 48-cell network with a multispot-beam antenna.

In case of MSSs, a single cell on the earth is the footprint of an antenna spot-beam from a satellite. If the satellite orbit is not geostationary, many satellites are involved in covering the same area all the day.

A handover procedure represents the automatic (i.e. 
seamless) rerouting of the radio portion of a call owing to signal quality, traffic management needs, or other reasons. In our analysis, we consider only the case of handover requests produced by the relative motion among satellites and mobiles, i.e. when a mobile moves from a coverage area (spot-beam footprint) to another coverage area. In an LEOMSS, handover requests are almost exclusively due to the orbital motion of satellites rather than to the motion of users or to the earth rotation motion [7].

Terrestrial microcellular systems and LEOMSSs will be characterised by very high time and space variations of traffic demand and a very high number of handover requests during the call lifetime. Therefore, a heavy amount of signalling will be managed by network entities.

In this paper, a handover request procedure is considered which is based on the signal quality received by the mobile user. Accordingly to a decentralised implementation, the handover procedure is 'mobile controlled': each active mobile subscriber (MS) continuously checks the power level $p$ reccived from the spot beam currently managing it and compares this level with that of neighbouring beams $p_{i}$. A handover request is issued by the MS to the satellite (currently managing it) whenever $p<p_{i}-h(h=$ hysteresis level) and $p$ falls below a given threshold $T$ [8]. If a handover procedure cannot be completed the communication is dropped and lost.

The handover from beam to beam within the coverage of a satellite is called 'interbeam handover', whereas it is called 'intersatellite handover' when it involves two spot-beams that belong to adjacent satellites. In this work, only intersatellite handovers are addressed.

A geometric constraint is considered to be fulfilled by any channel allocation in this work: two beams can reuse the same channel provided that their footprints are at least a distance $D$ away. $D$ is called the reuse distance [9]. The MSS under consideration is supposed to allocate channels by using a globally coordinated dynamic channel allocation (DCA). The DCA approach explained in Section 3 may temporarily allocate any channel to any beam on the basis of the fulfillment of the geometric criterion on the reuse distance and according to a suitable choice aimed to improve the channel utilisation. It is possible to implement a distributed DCA technique where each satellite with onboard processing capabilities allocates channels without involving earth stations: neighbouring satellites communicate channel status information via intersatellite links (ISLs), as in the Iridium system.

This work focuses on the definition of a mobility model suitable for LEOMSSs, on the study of channel rearrangement strategies to be used jointly with DCA, and on the comparison among various DCA solution and the fixed channel allocation (FCA) technique [Note 1] in terms of both blocking probabilities and signalling load per served call.

\section{Mobility mode}

To evaluate the impact of the handover management strategies on the performance of channel allocation techniques it is important to define a model according to which an MS with a call in progress may change the

Note 1: An FCA technique permanently allocates a fixed set of resources to each cell so as to fullill the requirement on the reuse distance [7] cell, i.e. a 'mobility model'. The coverage area has been divided into cells and each cell is illuminated by an antenna spot-beam from a satellite. All the cells irradiated by a satellite are disposed on the earth according to a hexagonal regular layout and have a circular shape obtained by means of beamforming that compensates the footprint distortion owing to the spherical nature of the earth surface (Fig. 1).

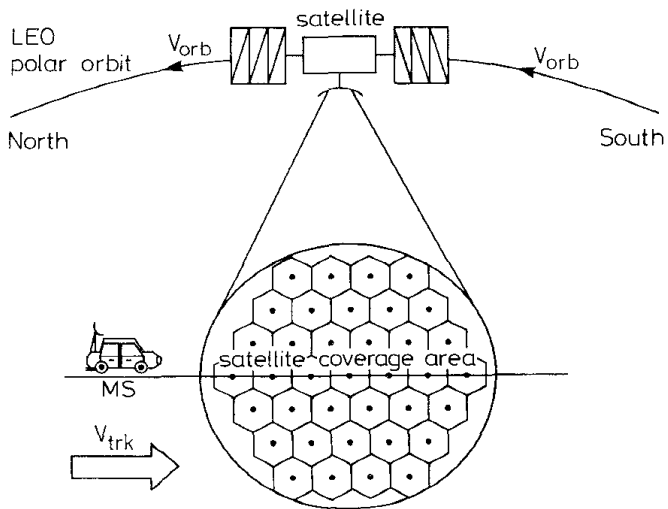

Fig. 1 Network of cells on earth iradiated by LEO satellite

The impact of handovers on the channel allocation performance depends on the orbit type chosen for the MSS (e.g. GEO, LEO). In the GEO case, the cells illuminated by a satellite are fixed (stationary) with respect to a point on the earth; then, handovers are produced only by the MS motion with respect to the earth. Moreover, the average call duration is negligible in comparison with the cell crossing times (with MS speed). This means that the handover occurrence is very low and, therefore, it can be considered, with a good approximation, that the user does not change cell (i.e. 'fixed user').

It has been shown in $[7,9]$ that a convenient representation of the LEO mobility scenario is that of considering any MS moving relatively to the footprints on the earth of the antenna beams with a speed equal to the ground-track speed of satellites $V_{t r k}$ (see Fig. 1). This approximation derives from the high value of $V_{i k k}$ $(\simeq 26,000 \mathrm{~km} / \mathrm{h}$ ) with respect to the other motion component speeds (i.e. the earth rotation around its axis and the user motion relative to the earth).

In defining the mobility model in the LEOMSS case, we have called 'source cell' the cell where the MS call begins and any cell reached by the relative MS during the call lifetime is named 'transit cell'. Moreover, in our simulations that are based on the mobility characteristics of the Iridium system, we have assumed that the cell side $R$ is equal to $212 \mathrm{~km}$ and the ground-track speed $V_{r m}$ is equal to $26,600 \mathrm{~km} / \mathrm{h}$.

In the LEO case, the following mobility assumptions have been made:

- MSs cross the cellular network with a relative velocity orthogonal to the side of the cells (Fig. 2).

- An MS crosses the cell at a random height $z$, which varies from $-R$ to $R$ (see Fig. 2).

- MSs cross cells at a constant velocity (relative to the satellite) equalling the satellite ground-track speed $V_{t r k}$.

- When a handover occurs the destination cell is the neighbouring cell in the direction of the satellite motion. 
- From the call outset in a cell an MS travels a distance (depending on $z$ ) defined as:

- uniformly distributed between 0 and $d(z)$, if the cell is the source cell for the call;

deterministically equal to $d(z)$, if the call is in a transit cell.

Where the distance $d(z)$ is derived according to Fig. 2 (see also the Appendix)

Fig.2 Mobility model assumptions and problem geometry in LEO case $d(z)=$ max. distance covered in cell by an MS from call arrival instant in this

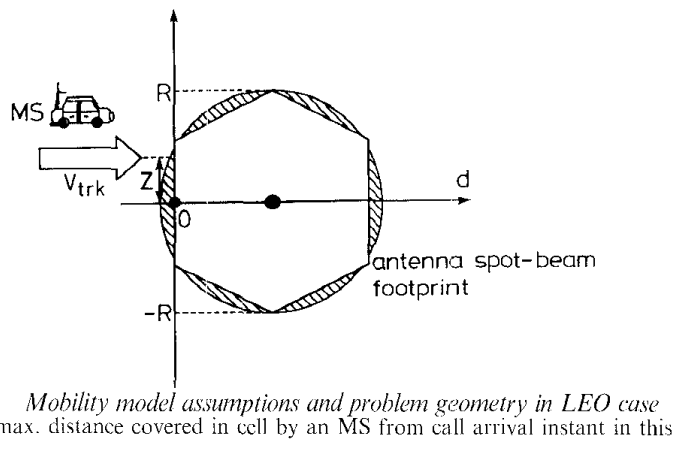

The user mobility will be characterised in this paper by the parameter $\alpha$ defined as

$$
\alpha \triangleq \frac{2 R}{V_{t r k} T_{m}}
$$

where $T_{m}$ represents the average call duration. Parameters $R$ and $V_{t r k}$ depend on the satellite constellation altitude; moreover, $R$ is also dependent on the half-power beamwidth (HPBW) of the spot-beams from the satellite antenna. By considering a fixed value for $T_{m}$, the MS mobility increases if $V_{t r k}$ increases and or $R$ decreases (i.e. $\alpha$ decreases). Further analytical details about this mobility model are given in the Appendix.

\section{Techniques to manage channel assignments in MSSs}

In this Section, a DCA technique is proposed that efficiently manages channel assignments in the cells of the network. Propagation and interference considerations have been simply represented by the constraint that if a channel is used in a given cell $x$, it cannot be reused in a given number $B$ of tiers of cells around $x$. These cells form the belt of interfering cells of $x, I(x)$. Then a same channel can be reused in different cells provided that they are separated by a distance $D$, the reuse distance. In this paper, we have considered $B=2$ (this is a value that could be attained by present technologies) and therefore $D=\sqrt{ }(21) R[7]$. Then the FCA reuse cluster is composed of seven cells [7].

Denote by $\Lambda(x)$ the set of available channels for cell $x$ at the call arrival instant in $x$, i.e. those channels not used both in $x$ and in $I(x)$. The proposed DCA technique is described in correspondence with three main events during the call lifetime: the call attempt, interbeam handovers, the call end. In particular, various alternative approaches are proposed to manage efficiently interbeam handovers.

\section{1 Management of new call attempts}

Assume that a new call arrival must be served in cell $x$ :

$\square$ if it results $\Lambda(x) \neq \varnothing$, the best channel to be allocated is selected on the basis of the evaluation of a cost function $C_{x}(i)$ for each channel $i$ belonging to $\Lambda(x)$ :

$$
C_{x}\left(i^{*}\right)=\min _{i \in \Lambda(x)}\left\{C_{x}(i)\right\}
$$

The cost function $C_{x}(i)$ is defined later in this Section. If more channels verify eqn. 2 , a random choice is performed to obtain channel $i^{*}$. Then, channel $i^{*}$ is allocated to cell $x$.

$\square$ If it results $\Lambda(x)=\varnothing$, the new call arrival in $x$ is blocked and lost.

The cost function $C_{x}(i)$ used in eqn. 2 is described subsequently. We start by considering the channels allocated according to FCA, assuming a reuse distance equal to $D$; let $F_{D}(x)$ be the set of channels allocated to cell $x$ by FCA. A fixed allocation assures a distribution of channels among the cells of the network with the minimum possible reuse distance, i.e. $D$. With the proposed allocation technique, we select channels belonging to $F_{D}(x)$, whenever possible, to serve a call in a cell $x$. Therefore the allocation cost contribution for channel $i \in \Lambda(x)$, owing to the interfering cell $k \in I(x)$, $C_{x}(k, i)$, can be expressed as

$$
C_{x}(k, i)=u_{k}(i)+2\left(1-q_{k}(i)\right), \quad \forall k \in I(x)
$$

where $u_{k}(i)$ and $q_{k}(i)$ are given by

$$
\begin{gathered}
u_{k}(i)= \begin{cases}1, & \text { if } i \in \Lambda(k) \\
0, & \text { otherwise }\end{cases} \\
q_{k}(i)= \begin{cases}0, & \text { if } i \in F_{D}(k) \\
1, & \text { otherwise }\end{cases}
\end{gathered}
$$

In defining the cost function contribution $C_{x}(k, i)$ relative to the status of channel $i$ in cell $k \in I(x)$, the first term is introduced to reuse the channel that results locked in the greatest number of interfering cells of $x$, whereas the second term tries to allocate in $x$, whenever possible, a channel belonging to the regular FCA pattern. In addition to this, we have chosen to weight this second contribution by a factor 2 to privilege a channel distribution among cells according to FCA. Therefore the overall cost function can be obtained as

$$
C_{x}(i) \triangleq q_{x}(i)+\sum_{k \in I(x)}\left\{C_{x}(k, i)\right\}, \quad \forall i \in \Lambda(x)
$$

where the term $q_{x}(i)$ is added in eqn. 6 to take into account that it is preferable to allocate in $x$ a channel $i$ belonging to the nominal channel set of $x$ (i.e. $i \in$ $\left.F_{D}(x)\right)$. Further details about this cost-function DCA technique and useful examples are given in [10].

\subsection{Management of call end}

To improve the performance of a DCA technique we propose that when a call end occurs in a cell $x$, the channel assignments in $x$ must be redefined so as to deallocate in $x$ the channel at maximum cost. The cost function to be used in this case is complementary to that defincd in the Section 3.1 to manage new call arrivals. Then if the most convenient channel is different from the channel to be released owing to the call termination, an intrabeam handover (also called channel rearrangement) is required. Without the use of such a technique for the de-allocation routine, the DCA performance significantly worsens especially in the presence of high mobility systems (e.g. LEOMSSs) $[10,11]$.

\subsection{Management of interbeam handover requests}

The channel allocation performance is strongly dependent on the handover management strategy, especially in the LEOMSS case, where the interbeam 
handover rate is extremely high; according to the mobility assumptions made, an interbeam handover request occurs every $\simeq 38 \mathrm{~s}$ in the Iridium mobility case under examination (see Section 8).

Assume that an active MS is approaching the border of its cell $x$ and is going into an adjacent cell $y$. In this new cell the MS must be provided with a new channel to carry on the communication; subsequently, the channel used in cell $x$ must be released by the MS. Four alternative approaches are below proposed to manage interbeam handover requests.

- An interbeam handover is managed as a call termination in the current cell $(x)$, according to the procedure described in Section 3.2, followed by a call arrival in the destination cell $(y)$, managed according to the procedure proposed in Section 3.1. In the DCA case, a channel rearrangement may be required in the current cell by the deallocation algorithm to pack as much as possible the use of channels in the network. In the following Sections, the dynamic (fixed) channel allocation technique with this policy for interbeam handover requests is designed simply by DCA (FCA). Note that no specific handover prioritisation is adopted in this case.

- An alternative approach privileges the service of interbeam handovers with respect to the service of new call arrivals and requires a DCA technique: if no channel is available in cell $y$ after the channel de-allocation in cell $x$, carried out according to the technique defined in Section 3.2, a different de-allocation is searched in cell $x$ to free a channel in cell $y$. If this search is successful, it is possible to serve the interbeam handover request that otherwise should be blocked. This interbeam handover strategy requires channel rearrangements ad hoc; the dynamic channel allocation technique with this policy for interbeam handover requests will be designated by 'DCA with rearrangements ad hoc'.

- A further improvement of the previous interbeam handover strategy can be the following: when in the destination cell $y$ there is no available channel a possible channel rearrangement is searched in an interfering cell of $y$ (not only in cell $x$ !) to free a channel in $y$. If this search is successful it is possible to serve the interbeam handover. Similar techniques have been proposed to improve the performance of dynamic channel allocations in terrestrial cellular networks and they are named 'persistent polite aggressive' (PPA) techniques [12]. The interest here is in their applicability in an MSS scenario to privilege the service of interbeam handovers. This technique is denoted by 'DCA PPA-H'.

- The fourth strategy to manage interbeam handover requests allows the queuing of handover requests $(\mathrm{QH})$ if there is no available channel in the destination cell of the MS. The queuing of handover requests is made possible by a certain degree of overlap among the footprints on the earth of adjacent beams. Assume that, due to beamforming, spot-beam footprints are disposed on the earth according to a hexagonal regular layout (side $R^{\prime}$ ) and have a circular coverage with radius $R$ (Fig. 3). Within an overlap area an MS can receive the signals at least from two adjacent beams. In the literature the possible values for the ratio $R / R^{\prime}$ range from 1 to 1.5 for terrestrial cellular systems [12]. Obviously, the greater this ratio is, the greater the overlap area extension is and then the better the performance of the queuing technique is. Here, a conservative choice has been used: it has been assumed $R / R^{\prime}=1$, that is the minimum possible extension for the overlap area (see Fig. 3).

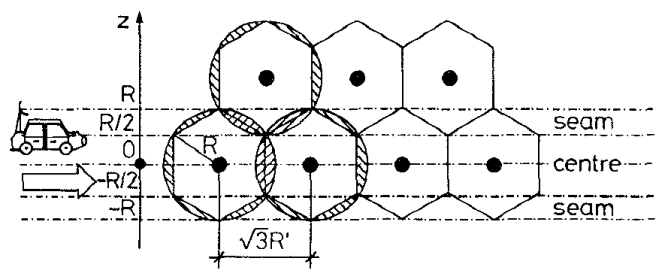

$$
\begin{aligned}
& \text { MII (i) } \\
& \square \text { (ii) } \\
& \text { QZय (iii) }
\end{aligned}
$$

Fig. 3 Overlap area assumptions

(i) circular coverage area for cell, with radius $R$

(ii) main coverage area for cell, with side $R^{\prime}=R$ (iii) overlap area between adjacent cells

The maximum value of the waiting time for a handover request $t_{\text {wmax }}$ can be derived as the time spent by the related MS to cross the overlap area with a speed $V_{i k}$. Of course, one must consider that the overlap area extension and then $t_{\text {wmax }}$ are random variables, because they depend on several parameters, such as the direction of MS (relative to the spot-beam footprint) motion, the satellite antenna characteristics and the propagation conditions. According to our mobility model $t_{\text {mmax }}$ is a random variable that only depends on the height $z$ of the MS crossing the cellular layout. The expected value of $t_{\text {wmax }}$ is equal to $7 \mathrm{~s}$ in the Iridium mobility case under examination (see Section 8 for an analytical evaluation of the expected value of $t_{\text {wmax }}$ ).

A handover request sent by an MS is served according to the following steps:

$\square$ if it results in $\Lambda(y) \neq \varnothing$, an arrival due to handover is served in cell $y$ and a call termination (Section 3.2) is performed in cell $x$

if it results in $\Lambda(y)=\varnothing$, the handover request is queued waiting for an available channel in cell $y$. In the meantime, the communication is served by the current cell.

We have assumed that the queue service discipline is of the FIFO type. A handover request leaves the queue owing to one of the three following reasons:

- The handover procedure is successful: the handover request is served (i.e. a channel becomes free in cell $y$ ) before the call is ended and its maximum queuing time has expired.

- The handover procedure has been useless: the associated call ends before the corresponding handover request is served and its maximum queuing time has expired.

- The handover procedure fails and the associated call is dropped: the handover has not been performed within $t_{\text {wrmax }}$ and the call is not ended before its maximum queuing time has expired.

The DCA (FCA) technique that allows the queuing of handover requests is denoted by DCA-QH (FCA-QH).

It is important to remark that the second and the third policies to manage interbeam handover requests are possible only jointly with a DCA technique where a channel rearrangement in a cell may affect the channel availability in interfering cells. 
The simulation results shown in Section 4 prove that - the first policy used jointly with DCA to manage interbeam handovers permits privilege implicitly in the service of interbeam handovers with respect to the service of new call arrivals

- the second policy attains a better handover prioritisation even if this improvement is slight

- the third solution achieves a good handover prioritisation and the signalling load increase owing to the PPA-H strategy is not heavy from low-to-medium traffic loads per cell (Fig. 9 in Section 4)

- the fourth approach gives the strongest prioritisation for the service of handover requests.

\section{Simulation results}

All the results shown in this Section were derived by computer simulations. The basic assumptions used are

- the call arrival process is Poisson-independent from cell to cell

- a uniform traffic in the cells of the network

- the call duration is exponentially distributed

- the simulated cellular network is parallelogram shaped and folded onto itself [11]

- in the case of interbeam handover strategies that allow the queuing of interbeam handover requests, the following additional assumptions have been made:

- minimum overlap area extension for spot-beam footprints disposed according to a hexagonal layout (conservative assumption)

-- infinite queue capacity

The values of the system parameters used in the simulations are summarised in Table 1.

Table 1: Values of parameters used in simulations

\begin{tabular}{lll}
\hline Symbol & Definition & $\begin{array}{l}\text { Value used in } \\
\text { simulations }\end{array}$ \\
\hline$\lambda$ & $\begin{array}{l}\text { average arrival rate for new } \\
\text { call attempts per cell } \\
\text { average call duration }\end{array}$ & 3 min \\
$T_{m}$ & $\begin{array}{l}\text { number of tiers in belt of } \\
\text { interfering cells for cell }\end{array}$ & 2 \\
$N$ & $\begin{array}{l}\text { number of cells per side in } \\
\text { simulated parallelogram- } \\
\text { shaped network } \\
\text { number of channels of } \\
\text { system }\end{array}$ & 7 \\
$M$ & mobility parameter & 0.32 in the Iridium case \\
& & $\rightarrow \infty$ in the GEO case \\
\hline
\end{tabular}

Denote by

$P_{h 1}$ the blocking probability for new call arrivals

$P_{b 2}$ the handover failure probability

$P_{n s}$ the probability of incompletely served call owing to the initial blocking or to the failure of a subsequent interbeam handover request

We have assumed that acceptable values for $P_{b 1}$ are of the order of $1 \%$, while the acceptable values for $P_{b 2}$ are at least two orders of magnitude smaller than the previous ones (i.e. $P_{b 2}=10^{-4}$ ). Note that the acceptable values for $P_{b 2}$ also depend on the degree of user mobility. For example, if the mobility increases (i.e. if $\alpha$ decreases), the number of handover procedures that a call in progress suffers during its lifetime $n_{h}$ [Note 2] increases and there is a probability $P_{b 2}$ that the call is dropped at each cell change. Then, assume that a given call dropping probability is acceptable from the user standpoint. Since a rough estimate of this dropping probability is given by the product $n_{h} P_{b 2}$ [7], we have severe requirements for $P_{b 2}$ if the mobility increases.

The comparison among DCA, DCA-QH, FCA, FCA-QH techniques in the Iridium mobility case (i.e. $\alpha$ $\simeq 0.32$ ) is shown in Figs. 4,5 and 6 for $P_{b 1}, P_{b 2}$ and $P_{n s}$, respectively. The following comments are in order.

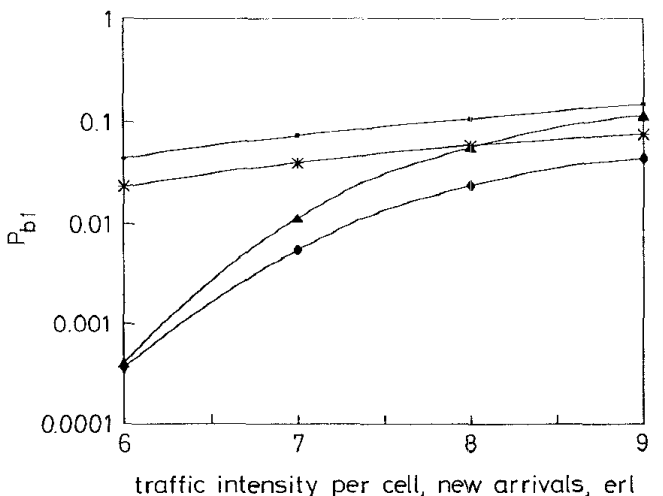

Fig.4 $P_{h}$ performance for DCA, DCA-QH, FCA, FCA-QH, for LEOMSS with $\alpha \simeq 0.32$ (ridium mobility case)

LEOMS WCA-QH

- $-\mathrm{FCA}$

DCA
- DCA-OH

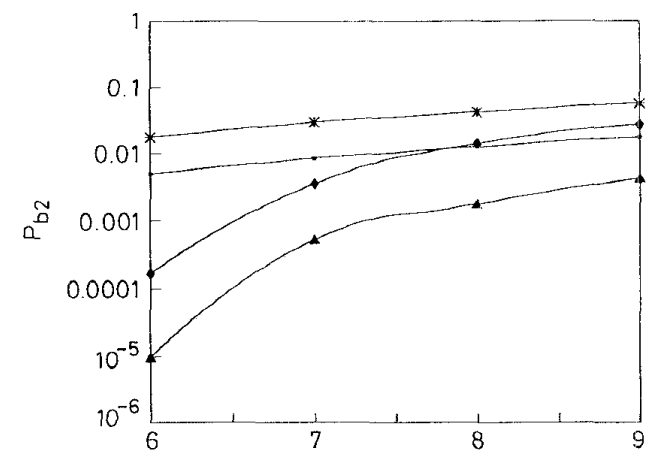

traffic intensity per cell, new arrivals erl

Fig. $5 P_{h 2}$ performance for $D C A, D C A-Q H, F C A, F C A-Q H$, for LEOMSS with $\alpha=0.32$ (Iridium mobility case)

- FCA-QH

* FCA

- DCA

The $P_{b 1}$ and $P_{b 2}$ performance of the FCA technique is unsatisfying because the values of $P_{b 1}$ and $P_{b_{2}}$ are too high all over the traffic range under examination. A way to improve the FCA performance is to allow the queuing of handover requests. FCA-QH permits to reduce $P_{b 2}$ of one order of magnitude with respect to FCA. Unfortunately, this decrease is obtained at the expenses of an increased value of $P_{b 1}$. The comparison between FCA and FCA-QH can be easily summarised by considering the only parameter $P_{n, s}$, as shown in Fig. 6.

DCA allows a significant performance improvement in terms of both $P_{b 1}$ and $P_{b 2}$ with respect to FCA. This

Note 2: In the Iridium mobility case, $n_{h}$ is of the order of 4.82 for an average call duration of 3 min. See the Appendix for an analytical cvaluation of $n_{i}$ 
comment applies also to the comparison between DCA and FCA-QH, but now the $P_{b 2}$ performance with DCA outperforms that one with FCA-QH only from low-tomedium traffic loads per cell.

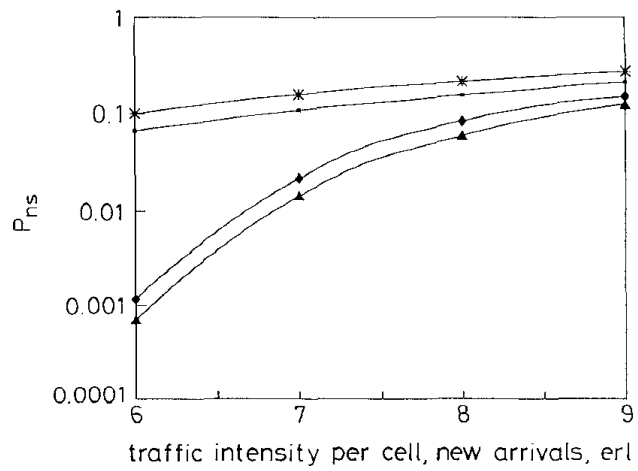

Fig. $6 P_{n \text { s }}$ performance for DCA, DCA-QH, FCA, FCA-QH, for LEOMSS with $\alpha \simeq 0.32$ (Iridium mobility case)

- FCA-QH

* $-\mathrm{FCA}$

$\longrightarrow$ DCA

- DCA-QH

Focus on the values of $P_{b 1}$ and $P_{b 2}$ obtained by DCA (see Figs. 4 and 5); it is interesting that $P_{b 2}$ values are significantly less than $P_{b 1}$ ones at the same traffic load and no specific handover prioritisation technique has been used. The proposed DCA technique reduces $P_{b_{2}}$ with respect to $P_{b 1}$ through the use of the de-allocation strategy described in Section 3.2. In other words, the proposed DCA technique gives an implicit prioritisation to the service of handover requests with respect to the service of new call arrivals.

However, the values of $P_{b 2}$ obtained through DCA do not meet the requirements for a great part of the traffic range under examination (see Fig. 5). Then, $P_{b 2}$ can be reduced by allowing the queuing of handover requests, i.e. we consider the DCA-QH technique. Now, the $P_{b 2}$ values are so reduced that we can also accept increased values of $P_{b 1}$ with respect to DCA. The advantages obtainable by the $\mathrm{QH}$ strategy are more evident in terms of $P_{n s}$, as shown in Fig. 6. Here, the techniques ranking in ascending order is FCA, FCA-QH, DCA, DCA-QH.

The behaviours of $P_{b 1}$ and $P_{b 2}$ for 'DCA with rearrangements $a d$ hoc' and DCA PPA-H techniques in the Iridium mobility case have not been shown and we limit ourselves only to qualitative considerations. Simulations have demonstrated that 'DCA with rearrangements ad hoc' as regards DCA allows little advantages in terms of $P_{b 2}$, at the expenses of a slight increase in $P_{b 1}$. Moreover, there is no significant difference in terms of $P_{n s}$ between these two techniques. Whereas DCA PPA-H permits to reduce significantly $P_{b 2}$; obviously, $P_{b 1}$ increases.

The comparison among DCA, 'DCA with rearrangements ad hoc' and DCA PPA-H techniques in the Iridium mobility case (i.e. $\alpha=0.32$ ) is shown in Fig. 7 in terms of $P_{n s}$. The advantages achievable by means of DCA PPA-H are evident: the $P_{n s}$ performance of DCA PPA-H is near to that one of DCA-QH; however, the latter technique attains the strongest prioritisation for the service of interbeam handover requests (i.e. the values of $P_{b 2}$ are more reduced with DCA-QH than with DCA PPA-H at the same traffic load). Nevertheless, DCA PPA-H seems to be an interesting alternative approach.
In Fig. 8, the performance of DCA and FCA techniques is shown in the GEOMSS case, where the users have been assumed fixed. Now, $P_{n s}=P_{b 1}$ because there is no handover procedure during the call lifetime (then, no interbeam handover strategy has been used). The values of $P_{n s}$ are much lower than those shown in Figs. 6 and 7 for the LEOMSS case at the same offered load per cell. This is due exclusively to mobility differences.

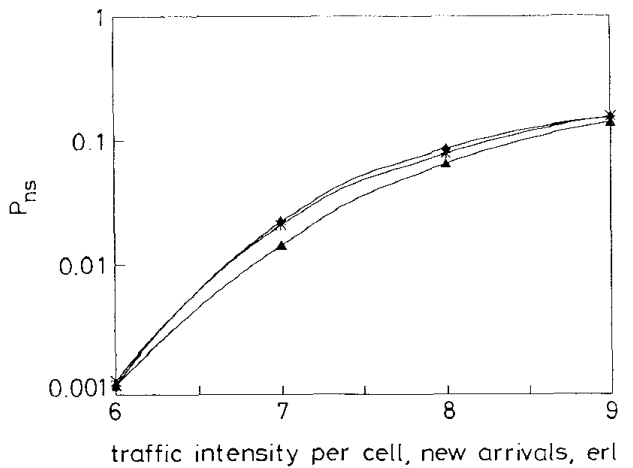

Fig.7 $P_{n z}$ performance for $D C A$. 'DCA with rearrangements ad hoc' DCA PPA-H, for LEOMSS with $\alpha=0.32$ (Iridium mobility case) * DCA rearrangements ad hoc

- DCA

- DCA PPA-H

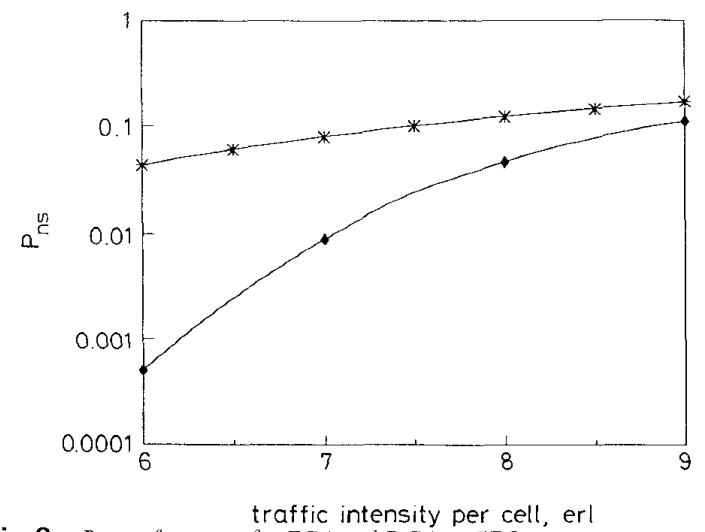

Fig. $8 P_{n s}$ performance for $F C A$ and $D C A$ in $G E O$ case - - DCA

Another parameter to be evaluated to investigate the practical feasibility of the proposed channel allocation techniques is the average signalling load to be carried out by the network per served call. In particular, we only take into account the signalling load per served call supported by the network to manage intrabeam ( 'channel rearrangement') and interbeam handover procedures and we denote this parameter by $\sigma$.

Table 2: Signalling load contributions due to intrabeam and interbeam handovers (number of signalling exchanges)

\begin{tabular}{ll}
\hline Events & $\begin{array}{l}\text { Contributions to signalling load } \\
\text { supported by network o }\end{array}$ \\
\hline Physical end & $a$ \\
Interbeam handover & $b$ \\
\hline
\end{tabular}

The events during the call lifetime that contribute to $\sigma$ and the related contributions evaluated in terms of 'number of signalling exchanges' are summarised in Table 2, taking into account the definition of the 
following parameters $a$ and $b$ :

$a \triangleq\left\{\begin{array}{l}1, \quad \text { if the channel de-allocation requires } \\ \text { a channel rearrangement } \\ 0, \quad \text { otherwise }\end{array}\right.$

$b \triangleq\left\{\begin{array}{l}4, \quad \text { if a channel rearrangement is performed } \\ \quad \text { in the source cell (de-allocation) and } \\ \quad \text { in another interfering cell (PPA-H) } \\ 3, \quad \text { if a channel rearrangement is only performed } \\ \quad \text { in the source cell (de-allocation) } \\ 2, \quad \text { otherwise }\end{array}\right.$

The signalling load contribution of each event during call lifetime represents the number of information exchanges between the mobile user and the satellite (according to the mobile controlled approach) that are needed to carry out the considered procedure.

According to the $\sigma$ contributions shown in Table 2, the average signalling load per served call $\sigma$ has been estimated as the ratio between the number of signalling exchanges during a given time interval in all the cells of the network and the number of served call in the same time.

The question to be addressed here is whether the DCA strategy with the PPA-H scheme to manage efficiently interbeam handovers causes a significant increase in signalling load owing to the channel rearrangements with respect to the simple DCA technique or the more efficient DCA-QH strategy. Towards this end, in Fig. 9 we compare the $\sigma$ performance for DCA, 'DCA with rearrangements ad hoc', DCA PPA-H and DCA-QH in the Iridium mobility case.

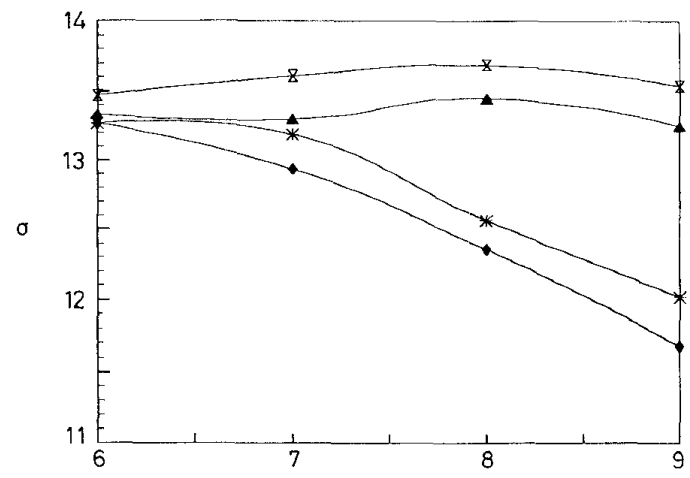

traffic intensity per cell, new arrivals, ert

Fig.9 operformance for the DCA techniques considered in LEOMSS, $\alpha$ $=0.32$

* - DCA rearrangements ad hoc

DCA

DCA PPA-H

$\times-D C A-Q H$

As for the DCA technique, $\sigma$ decreases if the traffic increases, because $P_{h 2}$ increases and then the average number of handover procedures per call decreases. 'DCA with rearrangements ad hoc' causes a slight increase in the signalling load with respect to DCA. Whereas DCA PPA-H increases the signalling load as the traffic load per cell is heavier; these high values of $\sigma$ are due to the additional channel rearrangements to serve the arrivals due to handovers. Finally, the DCA$\mathrm{QH}$ technique requires the heaviest signalling load $\sigma$ because it is the technique that greatly reduces the handover failure probability. Therefore a greater number of successful handover per served call is expected.
An interesting result is that DCA PPA-H technique does not increase $\sigma$ with respect to DCA-QH technique. Moreover, for low traffic loads per cell DCA, DCA-QH and DCA PPA-H techniques have almost the same $\sigma$ values.

Considering the GEO case (i.e. fixed users), the proposed DCA technique has $\sigma$ values equal to 0.6 . In this case, $\sigma$ is only due to the possible channel rearrangement at the end of each call. Therefore the difference is evident with respect to the Iridium case, where $\sigma=$ $13: 12$. In the presence of high mobility systems the network has to manage a lot of signalling only to carry out interbeam handovers caused by the relative motion of satellites.

\section{Conclusions}

Efficient DCA approaches suitable for applications in MSSs have been proposed to cope with the increasing need of mobile satellite communications. The scenario envisaged was GEO- and LEOMSSs. Particularly in the LEO case, interbeam handover requests are extremely frequent during the call lifetime and therefore it was assumed mandatory to use suitable handover management strategies in order to reduce the handover failure probability.

A user mobility model was developed to evaluate the impact of handover management strategies on the performance of channel allocation techniques.

Several interbeam handover strategies were investigated to find an efficient way to manage the user mobility both from the channel allocation and the signalling load standpoint.

As for the reduction of the handover failure probability, interesting approaches seem to be the DCA PPA-H technique that tries to find a suitable channel rearrangement to serve an interbeam handover request that otherwise should be blocked, and the DCA-QH solution that permits the queuing of handover requests.

Finally, the DCA-QH solution attains the best performance from the handover management standpoint, but DCA PPA-H allows a satisfactory level of interbeam handover prioritisation with a lower signalling load from low-to-medium traffic loads per cell.

\section{Acknowledgment}

This work was carried out within the framework of the SAINT project (RACE II mobile project line). The authors wish to thank Dr. Michel Mazella of Alcatel Espace, leader of the SAINT project (RACE 2117), for his useful comments and suggestions about this work.

\section{References}

1 HUBER, J.F.: 'System alternatives - satellites and network aspects'. Presented at the first European workshop on Mobile/personal satcoms, panel discussion B, Frascati, Italy, October 1994

2 HAAS, Z.: 'Mobile communication networks', Presented at IEEE Globecom 94 - tutorial 3, San Francisco, CA, November 1994

3 DEL RE, E.: 'Satellite system integrated with the terrestrial cellular network for mobile communications". ESA technical report, ESA STR-228, August 1989

4 DEL RE, E. 'Objectives and research activities of COST 227 project - Integrated space/terrestrial mobile networks'. Proceedings of RACE mobile telecommunications workshop, Amsterdam, The Netherlands, May 1994, pp. 590-597

5 GANZ, A., GONG, Y,, and LI, B.: 'Performance study of low earth-orbit satellite systems', IEEE Trans., 1994, COM-42, (2/3/ 4), pp. 1866-1871 
6 MARAL, G., DE RIDDER, J.-J., EVANS, B.G., and RICHHARIA, M.: 'Low earth orbit satellite systems for communications', Int. J. Sat. Commun., 1991, 9, pp. 209-225

7 DEL RE, E., FANTACCI, R., and GIAMBENE, G.: 'Efficient dynamic channel allocation techniques with handover queuing for mobile satellite networks', IEEE J. Sel. Areas Commun., 1995, 13, (2), pp. 397-405

8 LIN, Y.-B., MOHAN, S., and NOERPEL, A.: 'PCS channel assignment strategies for hand-off and initial access', IEEE Per, Commun. 1994, pp, 47-56

9 DEL, RE E., FANTACCI, R., and GIAMBENE, G.: 'Performance analysis of a dynamic channel allocation technique for satellite mobile cellular networks', Int. J. Sat. Commun., 1994, 12, pp. $25-32$

10 DEL RE, E., FANTACCI, R., and GIAMBENE, G.: 'Handover and dynamic channel allocation techniques in mobile cellular networks', IEEE Trans., 1995, VT-44, (2), pp. 229-237

11 DEL RE, E., FANTACCI, R., and GIAMBENE, G.: 'An efficient technique for dynamically allocating channcls in satellite cellular networks'. Proceedings of IEEE Globecom'95, Singapore, November 1995, pp. $1624-1628$

12 CIMINI, L.J., FOSCHINI, G.J., I, C.-L., and MILJANIC, Z.: 'Call blocking performance for dynamic channel allocation in microcells', IEEE Trans., 1994, COM-42, (8), pp. 2600-2607

13 CHU, T.-P., and RAPPAPORT, S.S.: 'Overlapping coverage and channel rearrangement in microcellular communication systems'. Proceedings of Globecom'94, San Francisco, CA, NovemberDecember 1994, pp. 1674-1678

14 LIN, Y.-B., CHANG, L.-F, and NOERPEL, A.: 'Modeling hierarchical microcell/macrocell PCS architecture'. Proceedings of IEEE ICC'95, Seattle, WA, June 1995, pp. 405-409

15 DEL RE, E., FANTACCI, R., and GIAMBENE, G.: 'Different queuing policies for handover requests in low earth orbit mobile satellite systems', submitted to IEEE Trans. Vehicular Technol

\section{Appendix: Analytical considerations on the mobility model}

Refer to the situation shown in Fig. 2. The height according to which an MS crosses the cellular layout $z$ is assumed to have a uniform distribution from $-R$ to $+R$, whose probability density function $(p d f)$ is expressed as follows:

$$
p d f(z)=\frac{u[z+R]-u[z-R]}{2 R}
$$

where function $u(z)$ is given by

$$
u(z)= \begin{cases}1, & z \geq 0 \\ 0, & \text { otherwise }\end{cases}
$$

Once the height $z$ according to which an MS crosses a cell is defined (see Fig. 2), the related distance $d(z)$ crossed in the cell is

$$
d(z)= \begin{cases}\sqrt{3} R, & \text { if }|z| \leq \frac{R}{2} \\ 2 \sqrt{3}(R-|z|), & \text { if } R \geq|z|>\frac{R}{2}\end{cases}
$$

The average distance crossed in a cell by an MS results as

$$
E[d(z)]=\int_{-R}^{+R} d(z) p d f(z) d z=\frac{3 \sqrt{3}}{4} R
$$

Denote by $t_{s}$ the mobile residence time in a cell. $t_{s}$ is a random variable that can be derived as $d(z) / V_{t r k}$. Then the expected value of $t_{s}$ is given by

$$
E\left[t_{s}\right]=\frac{E[d(z)]}{V_{t r k}}=\frac{3 \sqrt{3}}{8} \alpha T_{m}
$$

It can be proved [14] that if $P_{b 1}=P_{h 2}=0, n_{h}$ (i.e. the average number of handover requests per call) can be obtained as follows:

$$
n_{h}=\frac{T_{m}}{E\left[t_{s}\right]}=\frac{8}{3 \sqrt{3} \alpha}
$$

According to the height $z$ of an MS in a cell, it crosses a distance $o(z)$ in the overlap area, whose expression is

$$
o(z)= \begin{cases}2 \sqrt{R^{2}-z^{2}}-\sqrt{3} R, & \text { if }|z| \leq \frac{R}{2} \\ \sqrt{R^{2}-z^{2}}-\frac{\sqrt{3}}{2} R & \\ +\sqrt{R^{2}-\left(|z|-\frac{3}{2} R\right)^{2}}, & \text { if } R \geq|z|>\frac{R}{2}\end{cases}
$$

To obtain the average value of $o(z)$ we have to use the $p d f$ of $z$ related to the source cell. In this case, if a spatially uniform traffic is assumed, the $p d f$ of $z$ is not uniform since the cell shape is hexagonal. We can demonstrate that the $p d f$ to be used for $z$ is the following trapezoidal function $p d f_{1}(z)[15]$ :

$$
p d f_{1}(z)=\frac{h(z)}{\frac{3 \sqrt{3}}{2} R^{2}}
$$

where $h(z)$ represents the distance crossed by the MS at the height $z$ in the curvilinear cell circumscribed to the hexagonal cell with side $R$ (see Fig. 3):

$$
h(z)=2 \sqrt{R^{2}-z^{2}}-o(z)
$$

Then, the average distance crossed in the overlap area is obtained as follows:

$$
E[o(z)]=\int_{-R}^{+R} o(z) p d f_{1}(z) d z=\sqrt{3} R \beta
$$

where

$$
\beta=\frac{4}{9}\left(\frac{\sqrt{3}}{3} \pi-\frac{3}{2}\right) \simeq 0.1394
$$

It is straightforward to note that the time spent by an MS to cross the overlap area is $t_{w \max }=o(z) / V_{t r k}$. Then the expected value of $t_{\text {wmax }}$ is given by

$$
E\left[t_{w \max }\right]=\frac{E[o(z)]}{V_{t r k}}=\frac{\sqrt{3}}{2} \alpha \beta T_{m}
$$

Finally, on the basis of the values used for $R, V_{t r k}$ and $T_{m}$ in the Iridium case, we have that

- $E\left[t_{s}\right] \simeq 38 \mathrm{~s}$

- $n_{h} \simeq 4.82$ handovers per call (without blocking)

- $E\left[t_{\text {wmax }}\right] \simeq 7 \mathrm{~s}$.

These are the values used in the text. 